Pensamiento Crítico Vol.17. N¹, pp. 67-78

\title{
Comportamiento de los tipos de cambio internacional y doméstico durante la inestabilidad financiera
}

\author{
Carlos Palomino Selem
}

\section{RESUMEN}

El comportamiento del tipo de cambio financiero doméstico (E) definido por la relación Nuevo Sol (PEN)/Dólares Americanos (USD), se encuentra determinado de forma significativa por el comportamiento que toma el USD, como divisa conductora, en el mercado financiero internacional expresado por la relación Euro (EUR)/USD.

Resulta importante señalar que la política cambiaria, dentro del esquema de política monetaria, dirigida por el Banco Central de Reserva del Perú (BCR), encuentra un factor de restricción notable para la administración del tipo de cambio.

Nuestra investigación concluye además, que el marco conceptual para entender los mercados financieros, y dentro de estos al mercado de divisa, se encuentra en el enfoque de activos o de portafolio de inversiones, donde los inversionistas se guían por las rentabilidades que ofrecen los activos dominados en dichas monedas; esta es una característica que viene tomando el comportamiento de los mercados, y que se ha pronunciado en el proceso de la crisis financiera que se viviera el año 2008 y, que por el ambiente de inestabilidad que se mantiene hasta la actualidad, la organización de los portafolios de inversión a nivel mundial vienen 


\section{Pensamiento Crítico Vol.I7. No I}

principalmente determinando el precio de la divisa; y aparece cuando el sistema financiero experimenta riesgos de liquidez; en estos momentos el dólar se aprecia respecto al resto de monedas.

Palabras claves: Mercado monetario, mercancia física, posición de compra, posición de venta, tasa de interés de los fondos federales, paridad de los tipos de interés, mercados emergentes.

\section{ABSTRACT}

In this paper we demonstrate that the performance of Peru's financial exchange rate is determinated significantly for the performance of USD like conducting currency in the international financial market

It is important to say that the policy of exchange rate policy in Perú into of a scheme Banco Central de Reserva del Perú's (BCRP) has a constraint from the outside, indirectly of Federal Reserve Bank in United State of American.

Our research concludes that the framework to learn the financial markets and particularly the currency markets is in the approach of assets portfolio, where investor make decisions looking for returns of these assets and their currencies. This characteristic has been deepen since financial crises in the 2008 and appears when the financial system experience liquidity risk. In this situation the USD be appreciated respect to rest of currencies.

Keywords: Money market, commodity, long position, short position, federal fund interest rate, party of interest rate, emerging markets. 


\section{Carlos Palomino Selem}

\section{Introducción}

Desde los últimos 12 años, aproximadamente desde 1995 a la fecha, los flujos de capitales internacionales han avanzado hacia la integración de los mercados en general, particularmente entre los mercados financieros y de commodities, así como entre los centros financieros y los mercados emergentes. La magnitud mundial de los mercados de acciones y bonos representa el doble del nivel del PBI, y la capitalización bursatil ascendió a los 38 billones de dólares US en el año 2005 frente a los \$45 billones en los mercados de bonos; asimismo, la inversión directa extranjera proveniente de países de mercados maduros se ha recuperado sustancialemnte (ver Finanzas \& Pazarbasioglu, Goswami \& Ree, 2007). En este contexto, los mercados emergentes se han convertido, también, en exportadores netos de capital financiero hacia los mercados financieros maduros (Lane 2006), esto se ha realizado particularmente con vigor en los países asiáticos y los países petroleros; esto se debe al cambio en la percepción de los inversionistas extranjeros a consecuencia de observar el fortalecimiento del sector bancario, la flexibilización cambiariay la mayor cobertura en Reservas Internacionales de los países emergentes (Byun \& Oswald, 2006).

De otra parte, los países de mercados emergentes han mostrado una tendencia a aumentar en sus portafolios los títulos de economía maduras en lugar de los de sus propios países (Aurélio 2006 y French \& Poterba, 1991).

Esta integración se ha pronunciado en el periodo de inestabilidad financiera, crisis y recesión, los capitales se desplazan de un mercado a otro con rapidez y reorganizan sus portafolios con bastante velocidad entre segmentos de mercado cuando un anuncio que afecta una variable fundamental se presenta al mercado.

Esto nos ha motivado a iniciar una investigación sobre relaciones entre variables macrofinancieras y del mercado de capitales cuyo marco tórico y modelización se basan en un enfoque de activos y portafolio como llamarían Krugman \& Obstfeld (2008).

La finalidad de nuestra investigación es doble; por un lado busca vincular la relación existente entre el mercado de divisa y el mercado bursátil; trabajos en esta dirección, es decir, que buscan vincular a los mercados de acciones y bonos con el mercado cambiario se han pubilicado por parte de instituciones como el NBER, realizado por Ehrmann, 


\section{Pensamiento Crítico Vol.17. No I}

Fratzscher y Rigobon (2005) en el cual los mencionados autores demuestran una relación dentro del mecanismo de transmisión entre los distintos mercados financieros y el de divisa para el caso de Estados Unidos de América USA y Europa, mostrando que dicha relación se presenta con mayor agudeza en los periodos de shock en el precio de los activos.

\section{Marco teórico e hipotesis}

\section{Las hipótesis}

El periodo previo al proceso de inestabilidad financiera que se sucede desde el segundo semestre del año 2007, se distingue porque los agentes económicos en general (esto incluye compañías de inversión y no financieras) han actuado siguiendo una estrategia entre especulativa y de protección; lo cual ha llevado a que se constituyan portafolios que, por un lado, contengan activos refugio como la liquidez expresada en términos de USD, el oro y los instrumentos del tesoro USA; y por otro lado, activos de mayor nivel de riesgo como las acciones, los commodities en metales y energéticos (el crudo) vinculados al nivel de actividad económica que experimenta la economía mundial.

Particularmente, cuando las expectativas sobre el nivel de actividad económica mejora, se deja en términos generales los activos refugio, principalmente USD entre los más vendidos ${ }^{1}$, lo cual hace que la divisa conductora pierda en términos cambiarios respecto al resto de divisas mayores y divisas de mercados emergentes incluido el PEN.

La política cambiaria del BCR cumple un rol de seguimiento y reacción frente a los efectos que trae la reorganización de los portafolios de los inversionistas internacionales; si estos últimos valorizan sus expectativas en términos alcistas y de recuperación económica, sus portafolios se encontrarán concentrados (largos ${ }^{2}$ ) en acciones y/o commodities, lo cual lleva a que se encuentren vendidos en USD; por lo que la depreciación del USD hace que el PEN se revalorice y el BCR intervenga de forma sostenida en el mercado de divisas.

1 Posición vendida (short), cuando un inversionista vende un instrumento sostenido en su cartera.

2 Largo o long, significa que los inversionistas han comprado y sostiene dichos activos en su portafolio. 


\section{Carlos Palomino Selem}

\section{El Marco teórico}

Los portafolios de los inversionistas internacionales se han constituido entre dos tipos de activos; estos son los activos refugio, y los activos de riesgo.

La mejora en las expectativas del nivel de actividad económica mundial y una política monetaria de dinero barato caracterizada por sostenimiento a niveles bastante bajos (entre $0 \%$ y $0.25 \%$ anual) de la tasa de interés de los fondos federales del Sistema de la Reserva Federal3 (SRF), cuya política la diseña e implementa el Comité Federal de Mercados Abiertos del SRF, conocido como FOMC por las siglas de su nombre en inglés, se expresaron en el comportamiento alcista de los índices bursátiles como el Índice Industrial Promedio Dow Jones (DJI) y el precio principalmente de los commodities como los metales base no ferrosos.

Esto trajo una organización de los portafolios de los inversionistas tomando posiciones largas en activos de mayor riesgo, dejando sus posiciones de liquidez en USD producto de la política de dinero barato (exceso de liquidez) y los menores rendimientos ofrecidos por las bajas tasas de interés remuneradas por los depósitos en USD. Por lo tanto, el USD se depreció frente al resto de monedas incluidas las divisas de los países emergentes de latinoamérica, particularmente del Nuevo Sol peruano.

Frente a cada movimiento de avance del DJI el USD responde depreciándose, lo cual trae como consecuencia apreciaciones contínuas en las monedas de los países emergentes, haciendo, como en el caso del Perú, que el BCR intervenga, reaccione, con distintas instrumentos de política monetaria y cambiara, entre ellas comprando USD en el mercado de divisas de Perú.

\section{Métodos y resultados}

En el discuro del método se empleará uno de tipo inductivo, porque de analizar de forma parcial la economía financiera de USA se realizará implicancias lógicas hacia la economía financiera de Perú en el plano monetario, cambiario y bursátil, para posteriormente llegar a una conclusión de un grado más general en la economía mundial.

3 Conocida como Tasa Fed. 


\section{Pensamiento Crítico Vol.I7. Nº I}

El trabajo analiza el comportamiento de las variables desde el periodo de inestabilidad financiera y presentación de la crisis de esta naturaleza, así como el de recesión económica, que se dió desde el segundo semestre del 2007 hasta el presente año 2009. Para ello, la información recogida sobre las variables de estudio ha sido diaria, en las estadísticas que así las publica; pero esto, también, ha obedecido a un fundamento metodológico, dado que se busca captar comportamientos de corto plazo que tiene como objetivo nuestra investigación.

Se empleará fuente de información secundaria para lo referente a las estadísticas tanto nacionales como internacionales, esta información se interrelacionará de forma descriptiva.

\section{Análisis y discusión}

La inestabilidad financiera cuyos efectos se observan en el DJI a inicios de julio del 2007 (ver Gráfico N. ${ }^{\circ}$ 1) alcanzó su nivel más crítico a inicios de marzo del 2009; la percepción de que la crisis empezó a ceder se consolida a inicios de julio del 2009 cuando la media móvil exponencial (EMA 50) atraviesa la línea de precios del DJI; periodo previo que entre marzo y julio refleja en general los buenos resultados económicos de las firmas corporativas USA y anuncios provenientes del calendario económico favorables.

Gráfico N. ${ }^{\circ} 1$

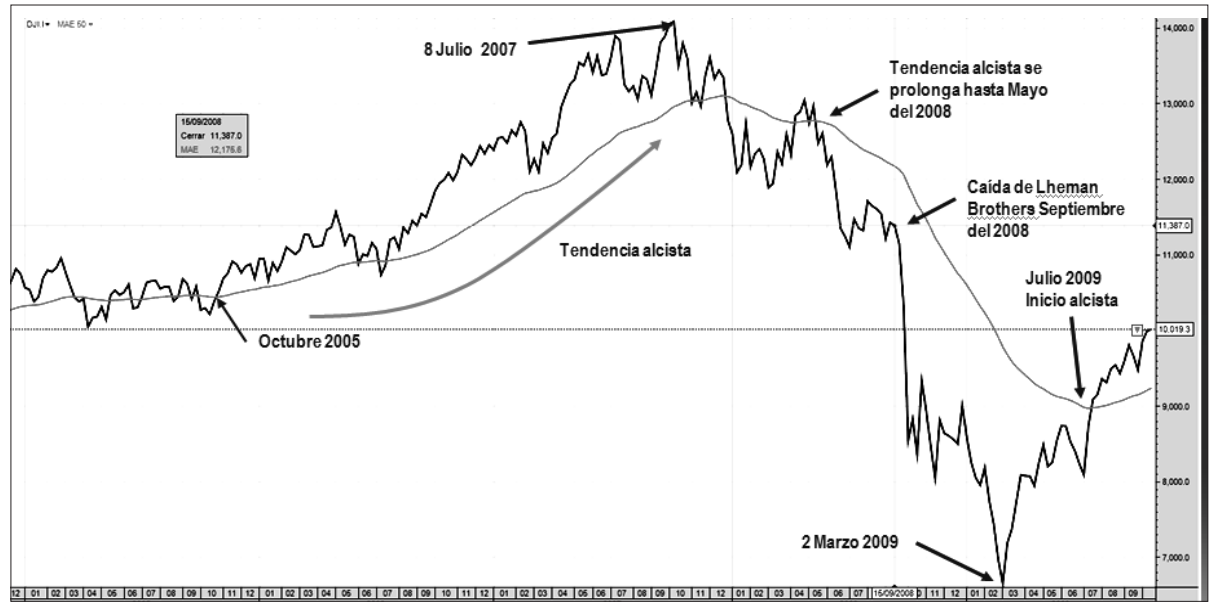




\section{Carlos Palomino Selem}

La relación entre el DJI y el nivel de actividad económica se puede establecer inicialmente inspirada por un Modelo de Arbitraje de Precios (APM) formulada por Ross (1976), en nuestro trabajo se expresa como:

$$
\begin{gathered}
\mathrm{R}_{\mathrm{DJl}}=\lambda(\Delta \mathrm{Y}, \mathrm{i}) \quad- \\
+-
\end{gathered}
$$

Siendo $\lambda$ una variable de sensibilidad del rendimiento del DJI $\left(\mathrm{R}_{\mathrm{DJI}}\right)$ respecto al crecimiento del nivel de actividad económica $(\Delta \mathrm{Y})$, "i" es la tasa de interés nominal.

En efecto, $\Delta \mathrm{Y}$ por encima de las expectativas del mercado $\Delta \mathrm{Y}^{\mathrm{EXP}}$, de modo que: $\Delta \mathrm{Y}-\Delta \mathrm{Y}^{\mathrm{EXP}}>0$ tendrán un efecto en $\mathrm{R}_{\mathrm{DJ}}$, esto se ha visto a partir del segundo trimestre del 2009 (véase Gráfico N.²).

\section{Gráfico N. ${ }^{\circ 2}$}

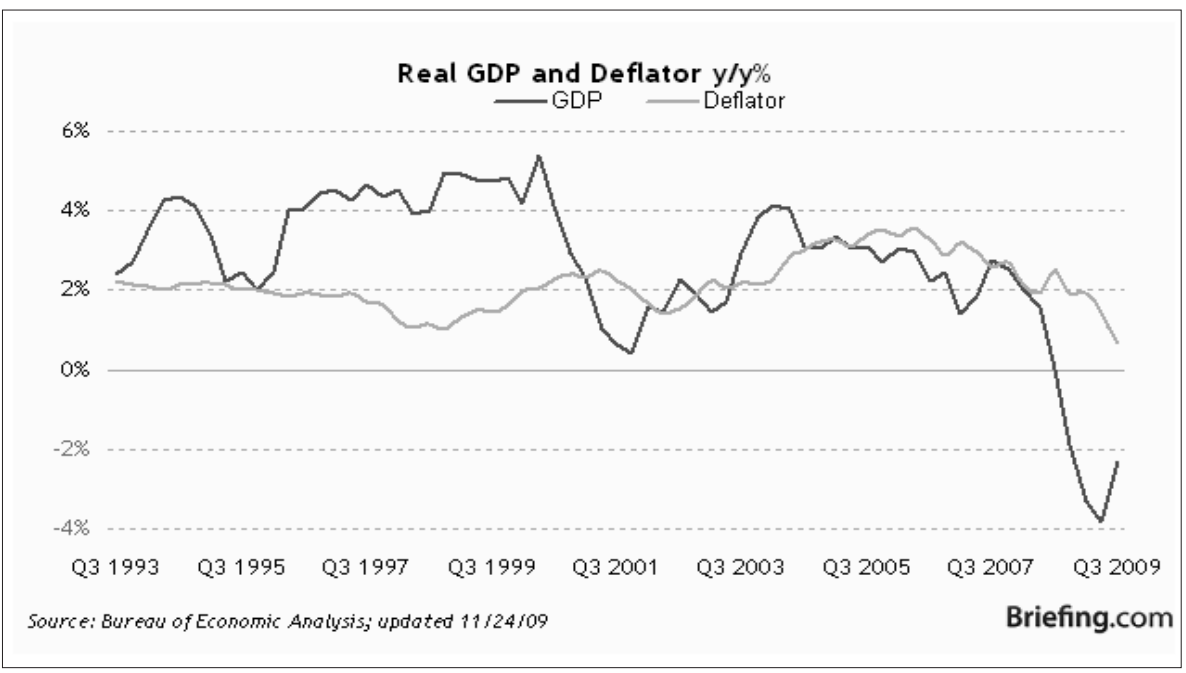

De otro lado, en el mercado monetario en USA, las políticas de dinero barato implementadas por el FOMC desde el segundo semestre del 2007 pero profundizadas a partir de septiembre del 2008, luego de la caída del banco de inversión Lheman Brothers, se constituyeron en uno de los fundamentos necesarios, más no suficientes, de la mejora del nivel de actividad en USA. 


\section{Pensamiento Crítico Vol.17. No I}

Si formalizamos lo dicho anteriormente, presentamos las relaciones siguientes:

$$
\mathrm{M}^{\circ}=\mathrm{M}_{\mathrm{d}}(\mathrm{i}, \mathrm{Y})
$$

Siendo $\mathrm{M}^{\circ}$ la oferta monetaria y $\mathrm{M}_{\mathrm{d}}$ la demanda de dinero que se encuentra en función de la tasa de interés nominal (i) e Y; de tal forma que el incremento de liquidez en $\Delta \mathrm{M}^{\circ}$ por la política de dinero barato implementada por el FOMC, vino acompañada en el periodo de inestabilidad financiera de una reducción importante en la tasa de interés de corto plazo, empezando con una reducción importante en la tasa Fed desde $5.25 \%$ hasta $0.25 \%$ y en la LIBOR a 3 meses (ver gráfica $N .{ }^{\circ} 3$ ), que se emplea para fines de capital de trabajo.

El apoyo del FOMC incrementando la base monetaria, y por ende la $\mathrm{M}^{\circ}$, con la intención de mantener las tasas de interés bajas estimuló mejoras en el Y, reduciendo la contracción de $\mathrm{Y}$, lo cual se puede entender como un $\Delta \mathrm{Y}$. Esto se puede ver en la figura N. ${ }^{\circ}$.

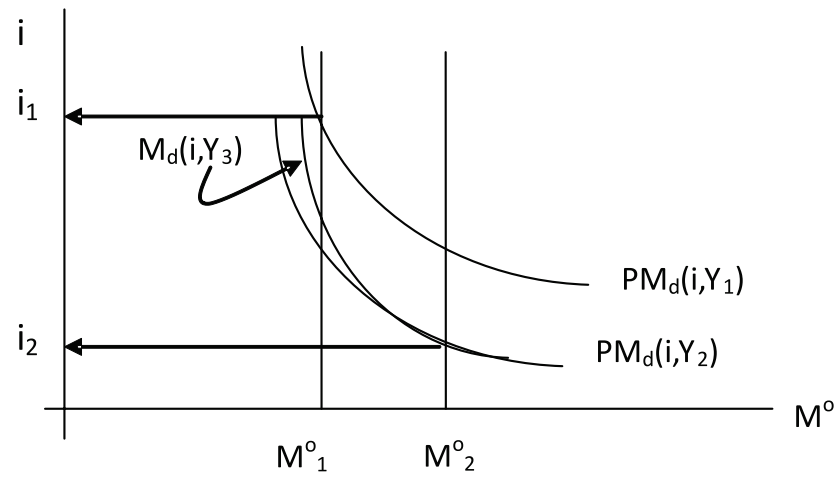

Figura N. ${ }^{\circ} 1$ : Efectos de la política monetaria sobre el nivel de actividad económica en el corto plazo

La reducción del nivel de actividad desde $\mathrm{Y}_{1}$ (momento previo a la recesión) hacia $\mathrm{Y}_{2}$ (nivel de piso alcanzado en el PBI) producto de la inestabilidad financiera que condujo a la recesión mundial que se inicia el año 2008, obligó a la política de dinero barato caracterizada por el $\Delta \mathrm{M}^{\circ}$ tal que $\mathrm{M}_{2}^{\circ}=\mathrm{M}^{\circ}{ }_{1}+\Delta \mathrm{M}^{\circ}$ y que se acompaña necesariamente con una reducción en la tasa de interés de referencia y del mercado secundario de $\mathrm{i}_{1}$ a $\mathrm{i}_{2}$, de tal forma que acompañada por la política fiscal hace que posteriormente, dentro del 


\section{Carlos Palomino Selem}

corto plazo, se recupere el nivel de actividad, reduciendo su contracción, pasando de $\mathrm{Y}_{2}$ a $\mathrm{Y}_{3}$, pero sin requerir de un ajuste hacia arriba de la tasa de interés.

La inestabilidad financiera y la política monetaria arrastró a una caída en el tipo de cambio financiero (nominal) E, Dólares Americanos/Euros (\$/€).

Si las expectativas sobre variaciones de la tasa de interés $\Delta \mathrm{i}^{\mathrm{EXP}}$ son nulas en el corto plazo, entonces las variaciones en el tipo de cambio E inició su depreciación que se dio desde septiembre del 2008 hasta marzo del 2009, luego de un proceso de consolidación de apreciación del USD como consecuencia la crisis financiera, la mayor desde 1929 (ver gráfica N. 4). En la figura N. 22 se esquematiza lo analizado.

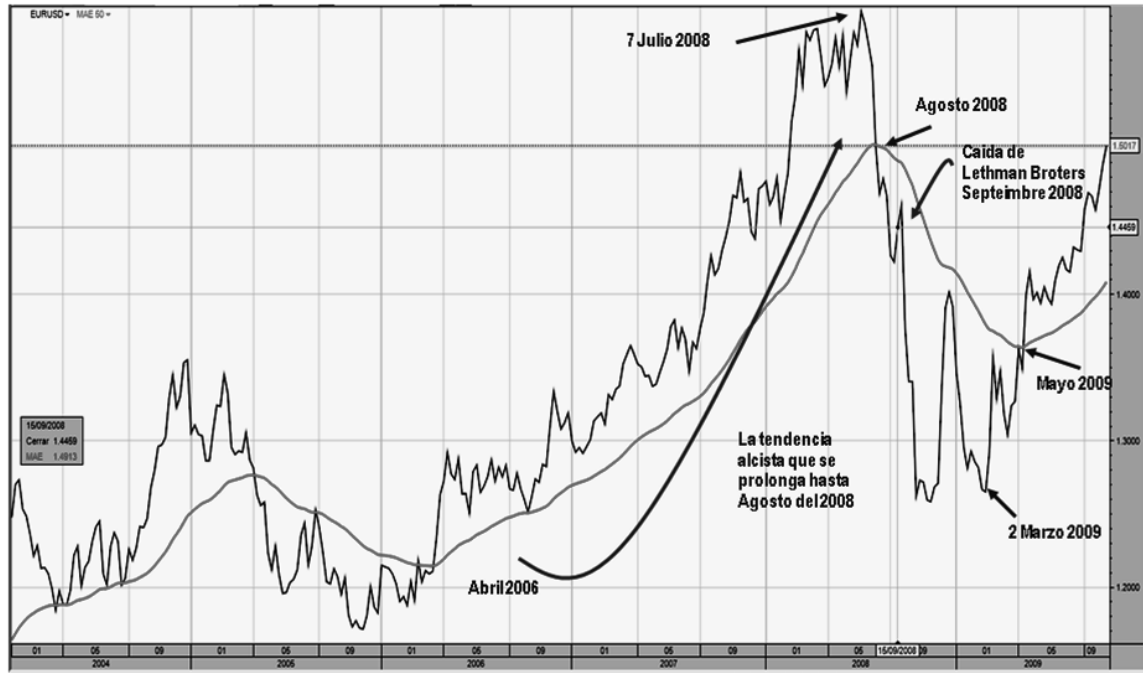

Bien, entonces el desempeño del tipo de cambio doméstico $\left(\mathrm{E}_{\mathrm{D}}\right)$ expresado por la relación Nuevo Sol (PEN) /USD, por efecto de cartera al depreciarse el USD inmediatamente el mencionado cruce $\mathrm{E}_{\mathrm{D}}$, también se reevaluaba al igual que el EUR. En efecto, la depreciación del USD hace que el rendimiento efectivo de los depósitos $e$ instrumentos nominados en USD pierdan atractivo, haciendo que los capitales especulativos se desplacen hacia instrumentos nominados en el resto de divisas, 
fortaleciendo a estas. La relación de paridad de los tipos de interés resulta en el modelo teórico esta afirmación:

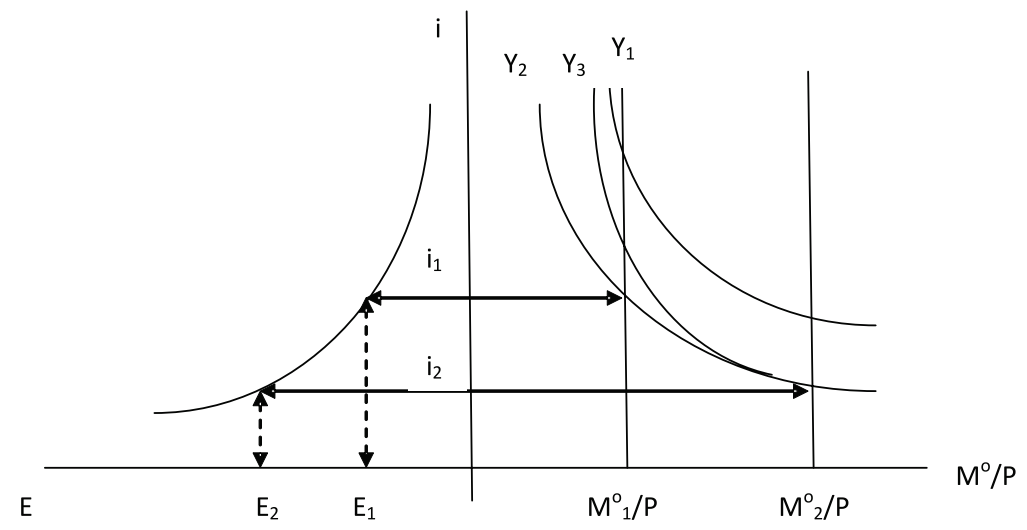

Figura N. 2: Relación entre el mercado monetario y cambiario

$$
\mathrm{R}=\mathrm{R}_{\mathrm{USD}}+\left[\mathrm{E}_{\mathrm{D} 2}-\mathrm{E}_{\mathrm{D} 1}\right] / \mathrm{E}_{\mathrm{D} 1}+\alpha--
$$

Siendo $\boldsymbol{R}$ el rendimiento en términos de moneda doméstica del $\boldsymbol{R}_{\mathrm{USD}}$, que sería el rendimiento de los instrumentos nominados en USD, y $\alpha^{4}$ la prima de riesgo por riesgo país en el caso de monedas emergentes. El $\alpha$ es la prima de riesgo adicional que exigiría un inversionista para tomar posiciones largas en la moneda doméstica de un país emergente. La ecuación de paridad de los tipos de interés expresa el rendimiento de un instrumento nominado en USD en términos de la moneda doméstica. Resulta pertinente mencionar que nuestro análisis se centra en el corto plazo y el rendimiento son para este plazo.

Si $e=\left[E_{D 2}-E_{D 1}\right] / E_{D 1}$, luego la expresión (3) se convierte en:

$$
\mathrm{R}=\mathrm{R}_{\mathrm{USD}}+e+\alpha---
$$

4 Para una formulación similar ver Parodi (2001). 


\section{Carlos Palomino Selem}

De la ecuación (3), si $\mathrm{E}_{\mathrm{D} 2}<\mathrm{E}_{\mathrm{D} 1}$, entonces $e<0$, hace que el rendimiento de los instrumentos en USD expresado en términos de moneda doméstica $R_{D}$ sea menor que el rendimiento de los instrumentos de la economía financiera doméstica:

$$
\mathrm{R}<\mathrm{R}_{\mathrm{D}}
$$

Por tanto, los inversionistas dejarán el USD haciendo mayor la diferencia entre $\mathrm{R}_{\mathrm{D}}$ - R. Esto explica la intervención del BCR para evitar una mayor reevaluación de la moneda doméstica.

De aquí se puede concluir que si $\mathrm{R}_{\mathrm{USD}}$ se mantiene bajo por efectos de la política monetaria de dinero barato donde $\Delta \mathrm{i}^{\mathrm{EXP}} \rightarrow 0$ y $\Delta \mathrm{Y}-\Delta \mathrm{Y}^{\mathrm{EXP}}>0$ el $\mathrm{R}_{\mathrm{DJI}}$ vendrá acompañado de e $<0$ como se parecía en la gráfica N. ${ }^{\circ} 5$.

\section{Conclusiones}

En el proceso de inestabilidad financiera desde fines del 2007 se observa que a la recuperación económica o los anuncios sobre fundamentos económico del calendario económico que alimentan expectativas de la recuperación del nivel de actividad, viene acompañado de una mejora en el comportamiento bursátil y un debilitamiento del USD arrastrando esto a una reevaluación del resto de monedas, incluyendo la denominadas divisas "duras" e incluso las monedas de países emergentes como el PEN. Lo cual resta autonomía a los BCR de los países como Perú para implementar una administración de los tipos de cambio.

\section{Referencias bibliográficas}

Aurélio, Marcel (2006); "Going Global: The Changing Patternof US Investment abroad" Federal Reserve Bank of Kansas Economic Review; tercer trimestre.

Blanchard, Oliver (2004), Macroeconomía; 2da. Edición, Editorial Pearson Prentice Hall, Madrid.

Ehrmann M., Fratzscher M. R. Rigobon (2005); "Stock, bonds, money markets an exchange rates: Measuring international financial transmission"; Working paper National Bureau Economic Research (NBER). 


\section{Pensamiento Crítico Vol.I7. No I}

French, K. \& James Poterba (1991); "Investor Diversification and International Equity Markets"; American Economic Review; Mayo.

Krugman, P. \& Maurice Obstfeld (2008); "Economía Internacional: teoría y política”, 7ma. Edición, Editorial Pearson. Madrid.

Lane, Philip (2006); "Global Bond Portfolio and EMU", International Journal of Central Banking; Junio.

Parodio, Carlos (2001); "Globalización y crisis financieras internacionales", Editorial Universidad del Pacífico, Lima.

Parzabasioglu, C., Goswani, M., Jack Ree (2007), "La Nueva Cara de los Inversionistas"; Finanzas \& Desarrollo, marzo.

Ross, Stephen (1976) "The Arbitrage Theory of Capital Asset Pricing"; Journal of Economic Theory; diciembre.

Páginas web: www.bcrp.gob, www.briefing.com, www.federalreserve.gov, www. Saxobank.com. 